\title{
Tricompartmental, Remote Intracerebral Hemorrhages Following Drainage of a Unilateral Chronic Subdural Hematoma: An Appraisal of the Pathogenesis
}

\author{
Prasad Krishnan ${ }^{1}$ Nabanita Ghosh ${ }^{2}$ \\ ${ }^{1}$ Department of Neurosurgery, National Neurosciences Centre, \\ Kolkata, West Bengal, India \\ 2Department of Neuroanesthesiology, National Neurosciences \\ Centre, Kolkata, West Bengal, India
}

\author{
Address for correspondence Prasad Krishnan, MS, MCh, \\ Department of Neurosurgery, National Neurosciences Centre, \\ Peerless Hospital Campus, 2nd Floor, Room No. 219, 360, \\ Panchasayar, Kolkata 700094, West Bengal, India \\ (e-mail: prasad.krishnan@rediffmail.com).
}

\begin{abstract}
Intracerebral hemorrhage is a rare and unanticipated complication after burr hole drainage of a chronic subdural hematoma and usually occurs on the same side as the

Keywords

- chronic subdural hematoma

- burr hole

- complications

- remote hemorrhage

- pathogenesis hematoma. In the absence of bleeding diathesis, iatrogenic injury or hypertension, it is commonly attributed to sudden expansion of the compressed brain, following rapid and uncontrolled removal of the subdural hematoma. Other factors like differential expansion of the various intracranial contents, abrupt changes in hemispheric balance, precipitous increase in focal cerebral blood flow, and unforeseen increase in cerebral venous pressure consequent to faulty positioning may also contribute to the pathogenesis of this complication.
\end{abstract}

\section{Introduction}

Burr hole evacuation of a chronic subdural hematoma is one of the most common neurosurgical procedures usually performed by trainees. While usually uneventful, one of the feared complications is a fresh intracranial hemorrhage which can be commonly attributed to intraoperative injury to the brain, postoperative hypertension, undetected secondary pathology like arteriovenous malformation, aneurysm, cavernomas or neoplasm, and preexisting coagulopathy. It may occur, however, in the absence of any of these pathologies.

\section{Case Report}

A 72-year-old diabetic, nonhypertensive male presented with progressive walking difficulty and inability to hold urine for a duration of 1 week. On examination, he had left-sided hemiparesis. Computed tomography (CT) scan of brain showed a right-sided hypodense chronic subdural hematoma (CSDH) with mass effect and midline shift ( - Fig. 1). Preoperative investigations including bleeding time, clotting time, prothrombin time, activated partial thromboplastin time and platelet counts were normal. There was no history of intake of anticoagulants or antiplatelet medications.

He underwent right frontal and parietal burr holes and drainage of the subdural hematoma under local anesthesia, but 4 hours postoperatively he became drowsy, was groaning, experienced localized pain and had decreased spontaneous movements of all four limbs. A repeat CT scan showed scattered intracerebral hemorrhages in both cerebellar hemispheres with left subependymal (abutting the frontal horn) and left occipital lobe bleed, right temporal hemorrhage as well as a small bleed in the right lateral ventricle and fourth ventricle ( - Fig. 2). Bleeding parameters were again repeated and found to be normal. As none of the hemorrhages were causing significant mass effect or midline shift, the patient was treated conservatively and recovered with residual cerebellar signs. published online

September 9, 2020
DOI https://doi.org/

$10.1055 / \mathrm{s}-0040-1713723$ ISSN 0973-0508.
(C)2020. Neurotrauma Society of India.

This is an open access article published by Thieme under the terms of the Creative Commons Attribution-NonDerivative-NonCommercial-License, permitting copying and reproduction so long as the original work is given appropriate credit. Contents may not be used for commercial purposes, or adapted, remixed, transformed or built upon. (https://creativecommons.org/licenses/by-nc-nd/4.0/).

Thieme Medical and Scientific Publishers Pvt. Ltd. A-12, 2nd Floor, Sector 2, Noida-201301 UP, India 


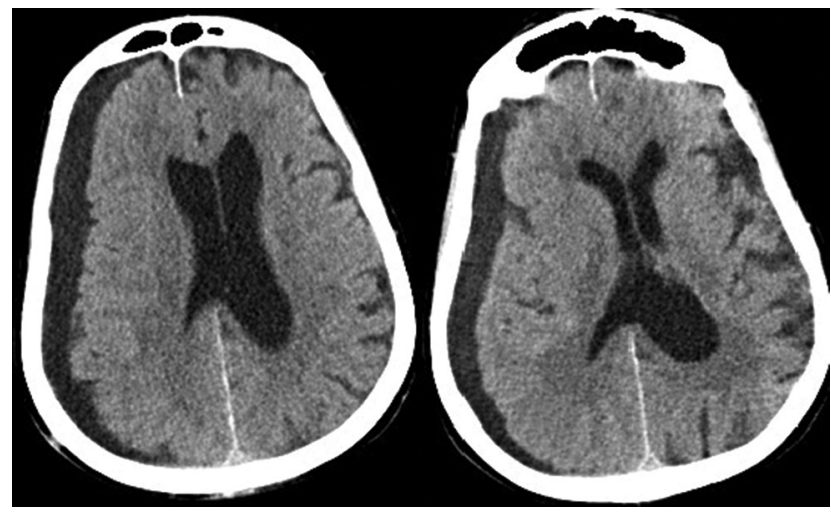

Fig. 1 Preoperative CT scan axial cuts showing right-sided hypodense chronic subdural hematoma with mass effect and midline shift.

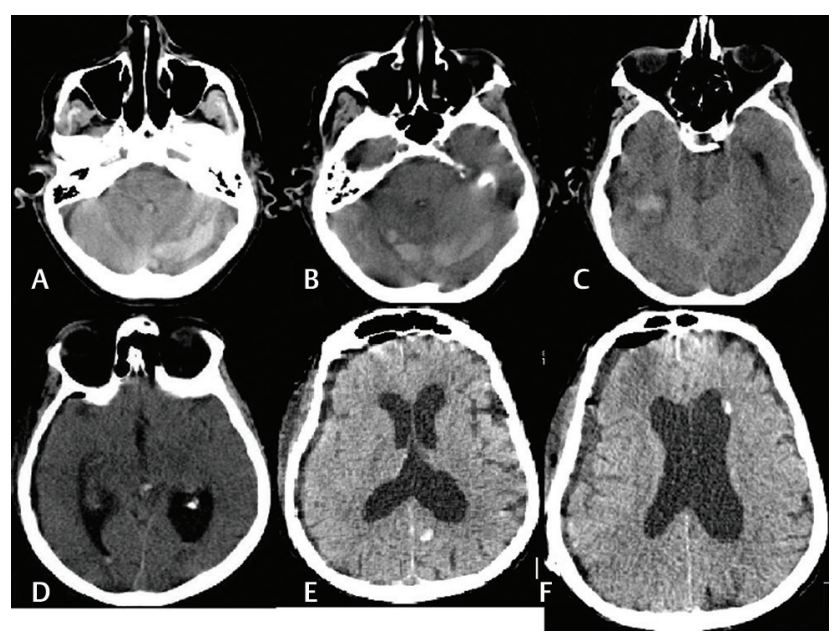

Fig. 2 Postoperative CT scans showing (A, B) bilateral intracerebellar and intra fourth ventricular hemorrhage, (C) right temporal intracerebral hemorrhage, (D) right lateral intraventricular hemorrhage, (E) left occipital hemorrhage, and (F) left frontal subependymal hemorrhage.

\section{Discussion}

The incidence of intracerebral hemorrhage (ICH) following evacuation of CSDH is reported to be 0.7 to $5 \%{ }^{1,2}$ It usually occurs on the same side as the $\mathrm{CSDH}^{3}$ but may rarely occur on the contralateral side or in the posterior fossa (cerebellum/ brain stem). The following theories have been postulated to explain the pathogenesis of ICH following evacuation of CSDH.

1. Vascular disturbances: Sudden escalation in blood flow due to rapid release of pressure during surgery might occur in the compressed hemisphere. This may result in bleed in an undetected contusion. ${ }^{3,4}$ Alternatively, cortical venous compression by the SDH may cause focal ischemia with dilatation of the proximal vascular bed, where capacity for cerebral autoregulation is lost..$^{2-4}$ Increased circulation after the local pressure is relieved in such a setting can lead to ICH. Sudden perioperative shift causing damage to cerebral vascular tree has also been hypothesized to be a cause of postoperative hemorrhage. ${ }^{4}$

2. Cerebral venous hypertension: Faulty positioning of the patient with hyperextension of the head and excessive rotation may lead to obstruction of the internal jugular vein and cause bleed due to increased cerebral venous pressure. $^{5}$

3. Differential expansion of intracranial contents: Deeper hemorrhages (subependymal or intraventricular) are explained by the varying elastic properties of neural tissue and cerebrospinal fluid due to which "mechanical stress" may occur at their interface as the brain expands. This may result in rupture of subependymal veins and cause distant bleed. ${ }^{2}$

4. Alteration of hemispheric balance: Drainage of CSDH may cause the contralateral compressed hemisphere to "fall towards" the falx with stretching and rupture or compromise in flow of contralateral cortical veins and remote site bleed. ${ }^{6}$ Increased mobility of intracranial structures (including posterior fossa structures) following drainage of CSDH has also been described. ${ }^{7,8}$ Transtentorial pressure gradient due to relative negative pressure in the supratentorial compartment at the time of drainage has been postulated to create a suction effect on the cerebellum with rupture of supracerebellar veins ${ }^{7,9}$ or transient occlusion of vermian veins ${ }^{5}$ and bleeding in the capillary bed.

A combination of these factors probably contributed to the remote, tricompartmental (i.e., on either side of the falx and in the posterior fossa) hemorrhages in our patient. A knowledge of the varied mechanisms leading to this complication will ensure implementation of preventive measures-careful evaluation of the CT scan preoperatively, proper positioning, slow controlled clot evacuation to avoid rapid intracranial pressure changes, and eschewing use of suction that creates negative pressure in the compartment being drained-and result in better outcomes.

\section{Funding}

None.

\section{Conflict of Interest}

None declared.

\section{References}

1 Ulivieri S, Oliveri G. Intracerebral haemorrhage following surgical evacuation of chronic subdural haematoma: case report. G Chir 2008;29(5):233-234

2 Savardekar AR, Salunke P. Subependymal hemorrhage following drainage of chronic subdural hematoma: Probable causative mechanisms and prevention strategies. J Neurosci Rural Pract 2015;6(2):252-254

3 Dinc C, Iplikcioglu AC, Bikmaz K, Navruz Y. Intracerebral haemorrhage occurring at remote site following evacuation of chronic subdural haematoma. Acta Neurochir (Wien) 2008;150(5):497-499, discussion 499 
4 Kim JK, Kim SW, Kim SH. Intracerebral hemorrhage following evacuation of a chronic subdural hematoma. J Korean Neurosurg Soc 2013;53(2):108-111

5 Chang SH, Yang SH, Son BC, Lee SW. Cerebellar hemorrhage after burr hole drainage of supratentorial chronic subdural hematoma. J Korean Neurosurg Soc 2009;46(6):592-595

6 Cohen-Gadol AA. Remote contralateral intraparenchymal hemorrhage after overdrainage of a chronic subdural hematoma. Int J Surg Case Rep 2013;4(10):834-836
7 Kollatos C, Konstantinou D, Raftopoulos S, et al. Cerebellar hemorrhage after supratentorial burr hole drainage of a chronic subdural hematoma. Hippokratia 2011;15(4):370-372

8 Rojas-Medina LM, Goel A. Brainstem hemorrhage secondary to evacuation of chronic subdural hematoma. Neurol India 2014;62(4):435-437

9 Amini A, Osborn AG, McCall TD, Couldwell WT. Remote cerebellar hemorrhage. AJNR Am J Neuroradiol 2006;27(2):387-390 